\title{
Performance Measurement of Vehicle Crash Imminent Braking Systems
}

\author{
Stanley Chien, Lingxi Li, and Yaobin Chen \\ Purdue School of Engineering and Technology \\ Indiana University - Purdue University Indianapolis
}

\begin{abstract}
As active safety systems have been introduced to passenger vehicles, there is an immediate need to develop a standardized testing protocol and scoring mechanism which enables an objective comparison between the performance of active safety systems implemented across various vehicle platforms. This project proposes a methodology for the establishment of such standards to evaluate and compare the performance of Crash Imminent Braking (CIB) systems. The proposed scoring mechanism is implemented based on track testing data in the evaluation of a 2011 model year passenger vehicle equipped with a CIB system.
\end{abstract}

